\title{
Andrea Gebhardt
}

\section{Auf dem Weg in die selbsternannte Mündigkeit}

\author{
Die Englische Schule zwischen Realismus, Neorealismus und Konstruktivismus
}

Die Forschungsschwerpunkte der Englischen Schule der internationalen Beziehungen (ES) haben sich bis heute immer wieder verlagert. Als die ES in den 50er Jahren mit dem British Committee begründet wurde, stand die historische Entwicklung von Staatensystemen im Mittelpunkt der Theorie. Danach prägte die Entwicklung des Konzepts der internationalen Gesellschaft die ES. Zentraler Bestandteil war die Unterscheidung von internationalem System und internationaler Gesellschaft sowie die Analyse von Institutionen internationaler Ordnung. In den letzten zwei Jahrzehnten verschob sich die Forschungsagenda von Betrachtungen hinsichtlich der europäischen internationalen Gesellschaft und ihrer Expansion bis hin zur globalen internationalen Gesellschaft und Weltgesellschaft. Es häuften sich Kontroversen zur exakten Mitgliederschaft der ES ${ }^{1}$ sowie zur Angemessenheit ihrer Bezeichnung. Zuletzt fand der Versuch statt, das theoretische Fundament der ES auszubauen und ihr einen Standort innerhalb der Theorien der internationalen Beziehungen (IB) zuzuweisen.

Die Theorie dieser Denkschule ist gekennzeichnet durch die Verregelung und Verrechtlichung der Staatenbeziehungen. Wie die Realisten gehen die Vertreter der ES von einem anarchischem Naturzustand der Staatenwelt aus. Dabei überwinden sie den Realismus und nähern sich dem Idealismus mit der Annahme, Anarchie könne durch eine fortschreitende Verregelung der internationalen Beziehungen überwunden werden, indem eine normative Integration von Teilbereichen des internationalen Systems geschaffen wird. Diese normative Integration erlaubt es, von der Herausbildung internationaler Gesellschaften zu sprechen.

Die Idee der internationalen Gesellschaft wird allgemein als wichtigster Beitrag der ES zur Disziplin der IB angesehen: Ihr Ausgangspunkt ist die Annahme, dass die Staaten im losen, ungeordneten internationalen System ihre eigenen Interessen verfolgen, während sie in einer Konstellation, in der sie an Normen und gegenseitige Abhängigkeiten gebunden sind, eine internationale Gesellschaft gestalten, in der sie ihre gegenseitigen Interessen respektieren. Solch eine Staatengesellschaft besteht, wenn eine Gruppe von Staaten sich aufgrund geteilter Interessen und Werte durch ein Regelsystem verbunden fühlt und über gemeinsame Institutionen verfügt ${ }^{2}$, wie

1 Die ES ist keine geschlossene Forschungsgruppe; zu ihren Vertretern werden Martin Wight, Herbert Butterfield, Adam Watson, Hedley Bull, Raymond J. Vincent und jüngere Wissenschaftler wie Richard Little, Barry Buzan, Nicholas J. Wheeler und Timothy Dunne gezählt.

2 Vgl. Hedley Bull, The Anarchical Society: A Study of Order in World Politics, 3. Aufl., London 2002, S. 13. 
z.B. die Diplomatie, das Völkerrecht, Regeln für den Kriegsfall oder internationale Organisationen. Die internationale Gesellschaft setzt das Vorhandensein eines internationalen Systems voraus. Dieses entsteht, wenn zwei oder mehrere Staaten sich untereinander derart beeinflussen, dass sie gegenseitigen Einfluss auf ihre Entscheidungen und Handlungen ausüben und sich dadurch veranlassen, sich wie ein Teil des Ganzen zu verhalten. ${ }^{3}$ Im engeren Sinne entspricht das Konzept des internationalen Systems dem anarchischen System der Realisten.

Die Vertreter der ES übernehmen vieles von den Realisten, insbesondere den Rückblick in die Geschichte sowie zu historischen Denkern. Die ES vertritt den interpretativen Ansatz mit einem derart ausgeprägten Historizismus, dass kaum eine andere Denkschule der IB an diesen heranreicht. Zugleich schafft sie Verbindungen zum Idealismus und versetzt sich somit in eine Mittlerposition zwischen idealistischer und realistischer Theorie. In den amerikanisch dominierten IB nimmt die ES nicht mehr als eine Randposition ein. Sie wird vielfach kritisiert, keine kohärente Theoriebildung zu betreiben und aufgrund ihrer Heterogenität keine geschlossene Forschungsgruppe zu sein.

Während die ES zu Beginn ihres Wirkens als eine Art realistischer Flügel betrachtet wurde, unternahmen Wissenschaftler in jüngerer Zeit wiederholt den Versuch, die ES mit anderen Theorien zu kreuzen. Verbindungen wurden zum klassischen Realismus, Konstruktivismus und Neorealismus gezogen, vereinzelt folgten Vergleiche zur Regimetheorie, Globalisierungstheorien sowie Theorien der Europäischen Integration. Hier reiht sich die vorliegende Untersuchung ein und schlägt eine Lösung dieser Standortsuche vor. Mithilfe der bisher getätigten Einordnungsversuche soll demonstriert werden, dass die ES mehr ist als ein bloßer Abklatsch des Realismus auf unserer Seite des Atlantiks.

\section{Die Englische Schule geht ibren eigenen Weg: Rationalismus als via media}

Fünf Kriterien grenzen die ES von anderen Ansätzen ab: Erstens, ihre Identifikation mit einer bestimmten Forschungstradition basierend auf einem geteilten Konsens an Kenntnissen und Ideen. Zweitens, das Bekenntnis zum interpretativen Ansatz zur Erforschung der internationalen Beziehungen. Drittens, die Überzeugung, die Theorie der IB muss normativ sein, entwickelt aus der Erkenntnis, dass die Staatengesellschaft Regeln und Normen hervorbringt, die im Mittelpunkt der Untersuchungen stehen sollten. Viertens, die internationale Gesellschaft als Merkmal der ES sowie fünftens, ihr Rationalismus als Mittelweg zwischen Realismus und Idealismus. ${ }^{4}$

Martin Wight, ein Gründungsmitglied der ES, unterteilte die politische Geistesgeschichte in die drei Rs - Realismus, Rationalismus und Revolutionismus ${ }^{5}$ - und

3 Vgl. ebd., S. 9.

4 Die ersten drei Kriterien wurden von Timothy Dunne übernommen. Vgl. Timothy Dunne, Inventing International Society. A History of the English School, London 1998, S. 6-11.

5 Revolutionismus kann mit Idealismus gleichgestellt werden. 
entwickelte mit der rationalistischen Tradition eine theoretische Grundlage für die ES und deren Konzept der internationalen Gesellschaft. Um den Realismus zu überwinden, beschäftigte er sich mit klassischen Ideen der europäischen Staatengesellschaft, insbesondere Schriften zum Völkerrecht, zum Gleichgewicht der Mächte und zur Diplomatie. Jenes Wissensgebiet war von der frühen Debatte zwischen Realismus und Idealismus noch unberührt geblieben. Diese Texte zu Recht und Ethik wurden von Wight als grotianische oder rationalistische Tradition identifiziert. ${ }^{6}$ Die Anziehungskraft des Rationalismus auf die ES liegt darin begründet, dass diese Tradition die überzeugendste Antwort auf die zentrale Frage der ES liefert:» What is international society? «

Aus Sicht des Realismus ist die internationale Gesellschaft ein Widerspruch in sich. In Abwesenheit eines zwischenstaatlichen Vertrages befinden sich die Staaten im vorgesellschaftlichen anarchischen Naturzustand, dem Kriegsstatus. Bezugnehmend auf die Thesen von Hobbes betrachten die Realisten internationale Beziehungen als permanentes Streben nach Macht. Frieden ist nicht mehr als das fragile Ergebnis gegenseitiger Unsicherheit und existentieller Abschreckung. Der Staat gilt als höchste Form politischer Autorität, seine Interessen schließen jegliche Beachtung der Interessen anderer Staaten aus. Die internationale Politik wird als immerwährendes Reich der Gewalt und des Überlebenskampfes beschrieben.

Dem Realismus entgegen steht der Revolutionismus. Während sich die Realisten auf die politischen Folgen der Anarchie konzentrieren, sind die Revolutionisten daran interessiert, eine moralische und kulturelle Ebene zu schaffen, die der Anarchie entgegenwirkt. Sie fordern eine internationale Gesellschaft der gesamten Menschheit, welche vielmehr eine Weltgemeinschaft als eine Weltgesellschaft sein soll. Außerdem beharren sie auf eine radikale Revision des negativen Menschenbilds der Realisten.

Der Rationalismus liegt zwischen diesen Extremen und fokussiert sich auf Diplomatie und Handel, in der Erwartung, dass solche Institutionen die Auswirkungen der Anarchie lindern. Seine Anhänger vertreten die Idee eines vorvertraglichen $\mathrm{Na}$ turzustands, der im Wesen weder chaotisch noch glückselig ist. Überdies ist der Mensch nicht als Geschöpf zu betrachten, dessen soziales Verhalten statischer und asozialer Natur ist, sondern als gesellschaftliches Wesen, das sich in kontinuierlicher Interaktion befindet. In der Evolutionsgeschichte erzeugte Verhaltensnormen beweisen, dass Gesellschaften durch wechselseitige Rechte und Pflichten der einzelnen Mitglieder geregelt werden. Folglich führt die Koexistenz souveräner Staaten sowie die Abwesenheit einer Weltregierung weder zu einem permanenten Kriegszustand noch zur Barriere der sozialen und wirtschaftlichen Zusammenarbeit.

Realismus und Revolutionismus sind nicht nur die Außengrenzen des rationalistischen Mittelwegs: Das rationalistische Bestreben nach internationaler Ordnung wird permanent durch die realistische Verfolgung des eigenen Interesses sowie der

6 Vgl. Martin Wight, International Theory: The Three Traditions, London 1991, S. 261.

7 Martin Wight, »An Anatomy of International Thought « in: Review of International Studies, 13 (1987), S. 222. 
revolutionistischen Suche nach transnationaler Gerechtigkeit unterminiert. Die rationalistische Tradition setzt die ES in eine konträre Position sowohl zum Realismus als auch zum Revolutionismus. Die Herausbildung von Rationalismus ist dabei als eine Art svia mediar, als dritter Weg zwischen zwei Extremen zu verstehen, der aus der Synthese der bisherigen Strömungen entstanden ist. ${ }^{8}$ Die Zusammenhänge der drei Theorietraditionen werden in folgender Tabelle verdeutlicht:

Die drei Theorietraditionen nach Wight

\begin{tabular}{|l|l|l|l|}
\hline & Realismus & Rationalismus & Revolutionismus \\
\hline $\begin{array}{l}\text { Ebene der } \\
\text { internationalen } \\
\text { Beziehungen }\end{array}$ & $\begin{array}{l}\text { Internationales } \\
\text { System }\end{array}$ & $\begin{array}{l}\text { Internationale } \\
\text { Gesellschaft }\end{array}$ & $\begin{array}{l}\text { Weltsystem/ } \\
\text { Weltgesellschaft }\end{array}$ \\
\hline $\begin{array}{l}\text { Historische } \\
\text { Vordenker }\end{array}$ & $\begin{array}{l}\text { Hobbes/ } \\
\text { Machiavelli }\end{array}$ & Grotius/Locke & Kant/Marx \\
\hline
\end{tabular}

Quelle: In Anlehnung an Jacek Czaputowicz, »The English School of International Relations and its Approach to European Integration« in: Studies E Analyses, 2, 2 (2003), S. 8.

\section{Die Englische Schule im Vergleich zu anderen Theorien der Internationalen Beziehungen - Versuch einer Einordnung}

Es waren amerikanische Autoren, die in der zweiten Hälfte des 20. Jahrhunderts den größten Beitrag zum Studium der IB leisteten. Dies trifft auf die bedeutendsten Strömungen zu und umfasst den Realismus, den Liberalismus, die Weltsystemtheorie sowie den Konstruktivismus. Die ES ist indessen eine Forschungsgruppe innerhalb der IB, die vielen unbekannt ist oder als Flügel des Realismus falsch interpretiert wird. Der Versuch, die ES in realistische Schuhe zu stecken, findet sich in den Texten verschiedenster Autoren wieder. ${ }^{9}$ Zugleich sträubt sich die ES selbst gegen eine feste Zuordnung und versucht sich als eigenständige Denkschule zu behaupten. Im Folgenden wird die ES mit dem klassischen Realismus, dem Neorealismus sowie

8 Vgl. Richard Little, »The English School's Contribution to the Study of International Relations « in: European Journal of International Relations, 6, 3 (2000), S. 398.

9 Vgl. Robert Gilpin, »The Global Political System« in: J. D. B. Miller / Raymond J. Vincent (Hg.), Order and Violence. Hedley Bull and International Relations, Oxford 1990, S. 120; Kalevi J. Holsti, The Dividing Discipline. Hegemony and Diversity in International Theory, London 1995, S. 33; Francis A. Beer / Robert Hariman (Hg.), Post-Realism. The Rhetorical Turn in International Relations, East Lansing 1996, S. 5. Radikalere Autoren betrachten die ES»as a Trojan horse for realism, a way of smuggling in power politics in a language of community«. Siehe Dunne, Inventing International Society, $\mathrm{aaO}$. (FN 4), S. 5. Andrew Linklater dagegen geht sensibler vor und erkennt zumindest den Unterschied zwischen dem Rationalismus der ES und dem Realismus an. Vgl. Andrew Linklater, Beyond Realism and Marxism. Critical Theory and International Relations, Basingstoke 1990, S. 10-21. 
dem Konstruktivismus verglichen. Ziel ist Gemeinsamkeiten und Unterschiede herauszustellen, um der ES auf dieser Basis einen eigenen Standort zuzuweisen.

\subsection{Englische Schule vs. Klassischer Realismus: Verbundenheit trotz Abnabelungsprozess}

Seit dem Zweiten Weltkrieg dominiert der von Hans J. Morgenthau begründete klassische Realismus die Theorien der IB. Jede andere Theorie der IB definiert sich anhand dieser Denkschule und steckt ihre Grenzen ihr gegenüber ab. Wenngleich der Realismus in den 30/40ern vor einem konkreten zeithistorischen Hintergrund entstanden ist, obliegt das realistische Denken einer langen geistesgeschichtlichen Tradition.

Der klassische Realismus ist eine Theorie, die auf empirischen Beobachtungen beruht. Dabei fokussiert er sich primär auf den Staat als moralische Gemeinschaft sowie auf das Verhalten seiner Regenten. Die Kunst der Staatsführung ist das Herzstück realistischen Denkens. Drei Grundannahmen Morgenthaus charakterisieren den klassischen Realismus: Erstens, die grundsätzliche wissenschaftliche Erklärbarkeit von Politik. Zweitens, die für das politische Geschehen zentrale Bedeutung der Kategorien Macht und Interesse. Drittens, die herausragende Rolle der Moral bei der Suche nach einer friedlichen und gerechten Welt. ${ }^{10}$

\subsubsection{Naturzustand / Menschenbild}

Aus Sicht der Realisten sind die fundamentalen menschlichen Bedürfnisse Sicherheit und Überleben. Diese entspringen einem pessimistischen Menschenbild, in dem der Einzelne von Machttrieb und Egoismus geleitet wird. Ähnlich wie der Idealismus, der vom wertorientierten Handeln der Individuen auf das Verhalten von Kollektiven schließt, überträgt Morgenthau seine anthropologische Prämisse auf das Verhalten von Staaten und geht von einem immerwährenden Konkurrenzkampf in der internationalen Politik aus. ${ }^{11}$ So wie Menschen in Abwesenheit einer Regierung in Anarchie leben, gilt dies für Staaten. Während Morgenthau die zerstörerischen Elemente des Menschen in dessen Natur begründet sieht ${ }^{12}$, stellt Reinhold Niebuhr die These auf, dass der menschliche Altruismus erst dann in Egoismus und Aggressivität umschlage, wenn sich die Menschen kollektiv organisieren und zusammenschließen. ${ }^{13}$ Die Ausführungen der ES stehen eindeutig im Widerspruch zu den An-

10 Vgl. Hans Joachim Morgenthau, Macht und Frieden. Grundlegung einer Theorie der internationalen Politik, 4. Aufl., Gütersloh 1963, S. 49-60.

11 Vgl. Hans Joachim Morgenthau, Scientific Man vs. Power Politics, 5. Aufl., Chicago/ London 1965, S. 198.

12 Hier folgt Morgenthau Hobbes, der den Machttrieb als Wesensmerkmal der Menschen bereits im >Leviathan< definiert hat. Vgl. Thomas Hobbes, Leviathan, Hamburg 1996, S. 81.

13 Vgl. Reinhold Niebuhr, Moral Man and Immoral Society. A Study in Ethics and Politics, New York 1932, S. 83. 
sichten Niebuhrs, geht die ES doch davon aus, dass die Staaten bzw. Individuen durch gemeinsame Normen und die daraus folgende Vergesellschaftung besänftigt werden. Hinsichtlich der Übertragung von Anarchie unter Individuen auf die zwischenstaatliche Ebene folgt die ES dem Analogieschluss Morgenthaus. In Bezug auf das Menschenbild überwindet die ES das pessimistische Bild der Realisten: Sie bestätigt einerseits den angeborenen Machttrieb, andererseits berufen sie sich auf die Vernunft des Menschen, welche über das egoistische Eigeninteresse siegt.

\subsubsection{Form der Internationalen Beziebungen}

Der klassische Realismus geht von der Koexistenz von internationalem System und Staatengesellschaft aus, zieht dabei aber nicht eine solch scharfe Trennlinie wie die ES. Das Konzept der Weltgesellschaft nimmt eine Schlüsselposition in Morgenthaus Diskussion der Formation einer Weltgemeinschaft ein. ${ }^{14}$ Morgenthaus Sicht der internationalen Politik beruht jedoch aufgrund seines pessimistischen Menschenbildes auf der Vorstellung eines anarchischen internationalen Systems. Dies beinhaltet, dass es keine zentrale Entscheidungs- und Sanktionsgewalt gibt. Hauptakteur der internationalen Politik ist somit der nach Macht strebende Nationalstaat, der seine eigenen Interessen gegen die Interessen anderer Staaten durchzusetzen versucht.

Die Ansichten des klassischen Realismus über die internationalen Beziehungen unterscheiden sich von denen der ES in Bezug auf das Mächtegleichgewicht, die Diplomatie sowie das Völkerrecht. Im Gegensatz zu den Vorstellungen der ES wird die Herstellung eines Mächtegleichgewichts aus Sicht der Realisten durch das System bedingt. Es entsteht automatisch, ist jedoch durch die permanente Machtmaximierung der Staaten in Gefahr sich aufzulösen. Die ES widerspricht dem, aus ihrer Sicht streben die Staaten zugunsten der Sicherheit bewusst ein Gleichgewicht an. ${ }^{15}$ Morgenthau weist dem Mächtegleichgewicht keine solch außerordentliche Funktion zur Erhaltung des Friedens zu, da die Vorstellung eines selbstregulativen Prozesses des Kräfteausgleichs in der internationalen Politik seiner Meinung nach einem veralteten Denken entspricht. Außerdem könne Macht nicht gemessen oder verglichen werden und der Versuch eines machtpolitischen Gleichgewichts ist daher von Anfang an zum Scheitern verurteilt. ${ }^{16}$

Das eindämmende Element der Macht ist für Morgenthau daher nicht das Gleichgewicht der Mächte; er setzt dem Prinzip der Machtpolitik Grenzen und räumt der Moral ihren Platz ein. Folgerichtig plädiert er für einen verantwortungs-

14 Vgl. Morgenthau, Macht und Frieden, aaO. (FN 10). Morgenthau charakterisiert die Weltgesellschaft als Vorbedingung für eine Weltregierung, welche die anarchische Struktur des internationalen Systems beenden könnte, glaubt jedoch zugleich, dass dies niemals eintreffen wird.

15 Vgl. Richard Little, "The English School vs. American Realism: a meeting of minds or divided by a common language? « in: Review of International Studies, 29, 3 (2003), S. 446.

16 Vgl. Morgenthau, Macht und Frieden, aaO. (FN 10), S. 146 und S. 179-180. 
bewussten Umgang mit der Macht und setzt - im Sinne von Max Weber - der idealistischen Gesinnungs- eine realistische Verantwortungsethik entgegen. Diese Verantwortungsethik manifestiert Morgenthau in der Methode des Friedens durch Ausgleich, dessen Instrument die Diplomatie ist. Er sieht in einer verantwortungsbewussten, moralisch begründeten Diplomatie den einzig effektiven Weg zur Bewahrung von Frieden und Stabilität in der internationalen Politik. Für die ES ist die Diplomatie ebenso eine der wesentlichen Institutionen zur Herstellung und Bewahrung internationaler Ordnung, jedoch ist ihre Rolle zur Friedensbewahrung weniger ausgeprägt als bei den klassischen Realisten.

Das Völkerrecht, das wiederum für die ES eine zentrale Funktion einnimmt, stellt für die Realisten lediglich einen sekundären Faktor dar. Morgenthau erkennt die Existenz des Völkerrechts zwar an, zweifelt aufgrund dessen Dezentralisation jedoch an der Wirksamkeit dieses Regulativs für den Machtkampfes in der internationalen Politik. ${ }^{17}$ John Herz dagegen räumt ein, das Völkerrecht sei sehr wohl in der Lage, Anarchie im internationalen System abzuschwächen und zu dämpfen. Er geht davon aus, dass Konflikte und militärische Auseinandersetzungen primär Resultate der anarchischen Struktur des internationalen Systems sind. ${ }^{18}$ Anders als Morgenthau identifiziert er damit das Problem von Krieg und Frieden nicht als anthropologisches, sondern als soziales. Konflikte ergeben sich demnach nicht aus dem angeborenen Machttrieb, sondern aus der Struktur des internationalen Systems. Die Gefahr in den zwischenstaatlichen Beziehungen resultiert gewissermaßen aus dem Fehlen einer zentralen Ordnungsinstanz. Dieses Dilemma könnte der Realismus durch Hinzuziehung des Konzepts der ES überwinden. Die internationale Gesellschaft geht über das internationale System hinaus und ebnet mit ihren fünf Ordnungsinstitutionen (Großmächte, Völkerrecht, Diplomatie, Mächtegleichgewicht und Krieg als letzte Instanz) die Bedingungen für ein friedliches Zusammenleben der Staaten und könnte auf diese Weise der realistischen Theorie einen wichtigen Input liefern.

\subsubsection{Macht und Interessen}

Die Instrumentarien der Macht nehmen im klassischen realistischen Denken eine zentrale Funktion ein. Obwohl Morgenthau einräumt, dass Staaten aus vielfältigen Motiven handeln können, bleibt das Mittel zur Verfolgung staatlicher Ziele jedoch immer die Macht. Diese hängt dabei eng mit dem nationalen Interesse der Staaten zusammen, welches sich wiederum daran orientiert, die Sicherheit und Machtposition der Staaten zu erhalten und zu verbessern und somit hauptsächlich der Macht dient. Bei der Verfolgung des Eigeninteresses handeln die Staaten rücksichtslos und setzen ihre Macht ausnahmslos zur Erlangung ihrer eigenen Ziele ein. Einen übergeordneten Gemeinwillen gibt es Morgenthau zufolge nicht, da die Menschen über

17 Morgenthau, Macht und Frieden, aaO. (FN 10), S. 246.

18 Vgl. John H. Herz, Politischer Realismus und Politischer Idealismus, Meisenheim am Glan 1959, S. 221. 
einen schmalen Unterbau an Gemeinsamkeiten hinaus, der auf der Essenz des menschlichen Daseins beruht, unter völlig verschiedenen Bedingungen leben und infolgedessen nicht die gleichen Interessen entwickeln. ${ }^{19}$ Hier trennen sich die Ansichten des klassischen Realismus von denen der ES, nach der es sehr wohl gemeinsame zwischenstaatliche Interessen und Prinzipien gibt, die weitreichende Kooperationen auf unterschiedlichen Ebenen ermöglichen. Darüber hinaus sollten die Realisten aus Sicht der ES die Rolle der Großmächte nicht nach dem Grad beurteilen, zu welchem diese schwächere Staaten mittels roher Gewalt zur Ordnung zwingen können, sondern nach dem Ausmaß, in dem sie von anderen Staaten als legitimierte Großmächte betrachtet werden. Macht bleibt auch für die ES zentral für die Analyse der internationalen Beziehungen, jedoch ist sie auch ein gesellschaftliches Attribut, das parallel zu anderen gesellschaftlichen Konzepten wie Prestige, Autorität und Legitimität untersucht werden sollte. ${ }^{20}$

\subsubsection{Methodologie}

An die Tradition von Machiavelli anknüpfend, betrachtet der Realismus Geschichte als Abfolge kausaler Zusammenhänge. Machiavelli geht davon aus, dass die Praxis die Theorie bestimmt und nicht umgekehrt. Das realistische Denken übernimmt diese Position und geht bei der Analyse der Politik nicht von der Wunschvorstellung einer Ordnung aus, sondern von den reellen Umständen des politischen Handelns. Ganz im Sinne von »theory meets history $\aleph^{21}$ knüpft die ES an diese >urrealistischer machiavellianische Ausgangsannahme an. Für die ES ist es die Geschichte, welche die Entwicklung der Theorien fördert.

Wie die Vertreter der ES gehören die klassischen Realisten zu den methodologischen Traditionalisten. Sie tragen die ältesten Traditionsgrundlagen außenpolitischen Denkens in sich und nehmen als Ausgangspunkt unter anderem Thukydides, Herodot, Polybios, Machiavelli und Hobbes. Analog zur ES gehen sie davon aus, dass internationales Verhalten durch gewohnheitsmäßig etablierte Normen reguliert wird.

Die Realisten begreifen die internationalen Beziehungen als Lehre der Außenpolitik von Staaten. Für sie sind die IB deckungsgleich mit der internationalen Politik und gehören folglich zum Bereich der politischen Wissenschaft. ${ }^{22}$ Aus Sicht der ES bestehen die IB aus verschiedenen Wissenschaften - der Politischen Wissenschaft, Wirtschaftswissenschaften, Rechtswissenschaften, Geschichte und Soziologie - und bilden somit ein eigenes Sachgebiet. Dazu stellte Wight folgende Überlegung auf:»If political theory is the tradition of speculation about the state, then international the-

19 Vgl. Morgenthau, Macht und Frieden, aaO. (FN 10), S. 233.

20 Vgl. Kai Alderson / Andrew Hurrell (Hg.): Hedley Bull on International Society, Basingstoke 2000, S. 24.

21 Barry Buzan / Richard Little, »The Idea of >International System tory « in: International Political Science Review, 15, 3 (1994), S. 231-255.

22 Hans Joachim Morgenthau, »The Nature and Limits of a Theory of International Relations « in: William T. R. Fox (Hg.), Theoretical Aspects of International Relations, Notre Dame 1959, S. 15. 
ory may be supposed to be a tradition of speculation about a society of states, or the family of nations, or the international community. ${ }^{23}$ Die ES beginnt nicht mit der Betrachtung des Staates und dessen Macht, wie dies die Realisten angehen würden, sondern nimmt als Ausgangspunkt die Untersuchung des Systems oder der Gesellschaft, in der die Staaten interagieren.

Der klassische Realismus ist in zweierlei Hinsicht anschlussfähig für die nachfolgende Theoriebildung: Ersteres betrifft das von den frühen Realisten betonte Spannungsverhältnis von Eigeninteresse und Moral. Allein die heutige Diskussion um die Rechtmäßigkeit sogenannter humanitärer Interventionen zeigt, dass Fragen der Moral nach wie vor bedeutsam für die internationalen Beziehungen sind. Aber auch Zweiteres spricht für den Realismus: Für die klassischen Realisten bedeutet das Nachdenken über Politik mehr als theoretisches Räsonieren. Sie ziehen ihre Erkenntnisse geradewegs aus empirischen Beobachtungen und lassen diese Einblicke wiederum in die praktische Politik einfließen. In dieser Nähe zum Gegenstand lassen sich Parallelen zur ES ziehen, die wie Morgenthau die reine naturwissenschaftliche Methode ablehnt und sich vielmehr auf Erfahrungswerte verlässt.

Die Konzeption der internationalen Gesellschaft entsprang der kritischen Auseinandersetzung der ES mit dem klassischen Realismus, da dieser die gesellschaftliche Natur des internationalen Lebens unterschätzt. Gleichwohl übernimmt die ES einige zentrale Anliegen der klassischen Realisten, insbesondere die Frage nach dem Verhältnis von Macht und Recht bzw. Macht und Ethik. Sogar die Schlüsselkonzepte der ES - internationales System, internationale Gesellschaft, Weltgesellschaft können bereits in den Werken klassischer Realisten identifiziert werden. Die Bedeutung der ES liegt folglich nicht in der Idee dieser Konzeptionen, sondern in ihrer Ausarbeitung.

Die ES litt lange darunter als»just another form of realism $\ll^{24}$ betrachtet $\mathrm{zu}$ werden, bis sie sich selbst vom puren Realismus abgrenzte. Dies soll nicht bedeuten, dass die ES alle Aspekte des Realismus zurückweist, sondern lediglich, dass die ES nicht identisch mit dem Realismus ist. Dennoch nimmt der Realismus eine zentrale Position in der ES ein, zu finden in den Werken von Edward Hallett Carr, Herbert Butterfield, Martin Wight und Hedley Bull. ${ }^{25}$ So hat die ES auf der einen Seite die Relevanz von Macht und Machtpolitik erkannt, doch»whilst international society might have to start with realism, it could never end with realism $\aleph^{26}$ : Der frühen rea-

23 Martin Wight, »Why is there no International Theory? « in: Herbert Butterfield / Martin Wight (Hg.), Diplomatic Investigations. Essays in the theory of International Politics, London 1966, S. 18.

24 Dunne, Inventing International Society, aaO. (FN 4), S. 3.

25 Siehe Edward Hallett Carr, The Twenty Years' Crisis 1919-1939: An Introduction to the Study of International Relations, 2. Aufl., London 1961; Herbert Butterfield, Christianity, Diplomacy and War, London 1953; Martin Wight, Machtpolitik, Bayreuth 1948 und Hedley Bull, The Control of the Arms Race. Disarmament und Arms Control in the Nuclear Age, London 1961.

26 Andrew Hurrell, »Society and Anarchy in International Relations « in: Barbara A. Roberson (Hg.), International Society and the Development of International Relations Theory, 2. Aufl., London 2002, S. 19. 
listischen Phase folgten eine rationalistische und zuletzt eine solidaristische Periode, in denen sich die ES nach und nach vom klassischen realistischen Denken befreit hat. Der Ausgangspunkt der ES wie der des Realismus war die Anarchie. Bereits hier entfernte sich die ES von der realistischen Theorie, indem sie Erklärungen dafür suchte, inwiefern Staaten ihren Machttrieb im Kontext von Anarchie kontrollieren können.

Laut Timothy Dunne gibt es zwei Argumente, aufgrund derer die ES als eine eigenständige Forschungsgruppe vom Realismus unterschieden werden muss: Zum einen die Tatsache, dass die Schule sich selbst als in einer postrealistischen Konversation befindlich betrachtet und zum anderen die Übereinstimmung innerhalb der ES, einen Ansatz zu verfolgen, der sich nicht mit herkömmlichen realistischen Theorien in einen Topf stecken lässt. ${ }^{27}$ João Marques de Almeida sieht in der Rückkehr zur Geschichte den entscheidenden Moment der ES, ihre Theorie auf eine neue Grundlage zu stellen und fügt hinzu, dass die ES und der Realismus auf völlig unterschiedlichen intellektuellen Traditionen beruhen. ${ }^{28}$ Das realistische Denken ist vorwiegend das Produkt einer im 19. Jahrhundert entstandenen Realpolitik, die als Gegenreaktion auf ältere internationale Theorien wie das Naturrecht, Liberalismus und die Tradition des Völkerrechts gilt. Dies sind genau jene Theorien, welche die ES wiederzubeleben beabsichtigt.

\subsection{Englische Schule vs. Neorealismus: Freunde oder Rivalen?}

Die Neorealisten reihen sich mit ihren Überlegungen in die Tradition der klassischen Realisten ein, gehen jedoch in Fragen der Theoriebildung über diese hinaus. Begründet wurde der Neorealismus 1979 von Kenneth Waltz mit seinem Werk »Theory of International Politics « ${ }^{29}$. Dieser war auf der Suche nach einer allgemeinen Theorie der IB, mit der sowohl Krieg als auch Phasen des Friedens in der internationalen Politik analysiert werden können. Dabei konzentrieren sich die Neorealisten wie die ES ausschließlich auf shigh politics - wie z.B. Sicherheitspolitik - und lassen soziale sowie ökonomische Aspekte weitgehend außer Acht. In seinen Untersuchungen geht der Neorealismus von drei Grundannahmen aus: Erstens, alle Staaten besitzen das zentrale Bedürfnis des Überlebens. Zweitens, bei der Umsetzung dieses Bedürfnisses handeln alle Staaten rational und drittens, Staaten können anhand ihrer Machtmittel unterschieden werden. ${ }^{30}$

27 Vgl. Dunne, Inventing International Society, aaO. (FN 4), S. 5.

28 Vgl. João Marques de Almeida, "Challenging Realism by Returning to History: The British Committee's Contribution to IR Forty Years On« presented at: BISA Annual Conference, Manchester, Dezember 1999, S. 1 und S. 3.

29 Kenneth N. Waltz, Theory of International Politics, Reading 1979.

30 Vgl. Niklas Schörnig, »Neorealismus « in: Siegfried Schieder / Manuela Spindler (Hg.), Theorien der Internationalen Beziehungen, Opladen 2003, S. 64-70. 


\subsubsection{Naturzustand / Menschenbild}

Wie die klassischen Realisten schätzt Waltz die menschliche Natur negativ ein. Demnach ist der Mensch egoistisch und aggressiv. Ansätze, denen ein positives Menschenbild zugrunde liegt, bezeichnet Waltz als naives Wunschdenken und gibt zahlreiche Beispiele dafür, dass die Menschen nicht per se gut und friedlich sind. ${ }^{31}$ Aufgrund dieses pessimistischen Menschenbildes, das sie auf die Staatenebene übertragen, sehen die Neorealisten wenige Möglichkeiten zum Zusammenspiel von Staaten, weil sie die internationalen Beziehungen als eine von Selbsterhaltungstrieb und Kooperationsverweigerung dominierte Sphäre begreifen. Da Anarchie herrscht, befinden sich die Staaten in permanenter Unsicherheit und müssen infolgedessen stets für den schlimmsten Fall - den Krieg - gewappnet sein.

Auch aus Sicht der ES liegt Krieg und Machtstreben in der Natur des Menschen begründet, jedoch vertritt die ES eine optimistischere Einschätzung als der Neorealismus in Bezug auf die Zähmbarkeit von Staaten und deren Bereitschaft, sich innerhalb der internationalen Gesellschaft Regeln und Bestimmungen unterzuordnen.

\subsubsection{Form der Internationalen Beziehungen}

Dem Konzept der internationalen Gesellschaft wie auch der Weltgesellschaft, wird von den Neorealisten kaum Beachtung geschenkt. Parallel zum Realismus bildet das internationale System die Ebene der internationalen Beziehungen. Dieses befindet sich in einem vorgesellschaftlichen anarchischen Zustand, welcher durch Hobbes' >state of war< gekennzeichnet ist. Durch Interaktion nähern sich die Staaten einander an und formen ein System. Neben dem Ordnungsprinzip, nach dem die Akteure im internationalen System anarchisch organisiert sind, unterscheidet Waltz zwei weitere Elemente, die zur Untersuchung der politischen Struktur des Staatensystems herangezogen werden: Ausgehend von der funktionalen Logik des Neorealismus fügen sich die Staaten in das Staatensystem ein und entwickeln dabei, trotz des Fehlens einer gemeinsamen Kultur, gemeinsame Regeln für ihre Interaktionen. Die Staaten werden dabei durch die Struktur des Staatensystems gelenkt und durch >strukturelle Kräfte $<$ zu einem gewissen Verhalten - z.B. zu einem Gleichgewicht der Mächte - gedrängt. ${ }^{32}$ Eine funktionale Differenzierung liegt vor, wenn die Akteure unterschiedliche Funktionen im System erfüllen. Waltz schließt aus der Annahme der Anarchie, dass die Staaten keine internationale Arbeitsteilung durchführen werden. Hier liegt ein wesentlicher Unterschied zur ES, nach der die Staaten bewusst eine internationale Gesellschaft formen, in der sie wechselseitig Aufgaben übernehmen.

31 Vgl. Kenneth N. Waltz, Man, the State and War. A Theoretical Analysis, New York 1959, S. 17-41.

32 Vgl. Richard Little, »Neorealism and the English School: A Methodological, Ontological and Theoretical Reassessment « in: European Journal of International Relations, 1, 1 (1995), S. 16. 
Das zweite Untersuchungselement bezieht sich auf die Machtverteilung zwischen den Akteuren. Für die Neorealisten sind drei Wege der Machtverteilung vorstellbar: Uni-, Bi- oder Multipolarität. ${ }^{33}$ Durch die theoretische Möglichkeit eines unipolaren Systems vervollständigt der Neorealismus die Gedanken des klassischen Realismus und der ES, die bisher von der Existenz mehrerer Großmächte innerhalb eines Staatensystems und somit von einem mindestens bipolaren System ausgingen. In Fortführung von Watsons mechanischem und Hedley Bulls zufälligem Mächtegleichgewicht, charakterisiert der Neorealismus das Gleichgewicht der Mächte»as an sunintended outcome of a system made up of states which, in theory, will be striving to survive «. ${ }^{34}$ Diese Auslegungen erscheinen zwar ähnlich, dennoch sind sie nicht identisch. Waltz geht davon aus, dass der Überlebensinstinkt der Staaten das Mächtegleichgewicht als dauerhaftes Kennzeichen des internationalen Systems sicherstellt und die Staaten sowohl mit der Kontinuität des anarchischen Systems als auch mit der Beibehaltung der vertrauten Struktur der internationalen Politik rechnen. ${ }^{35}$ Für die ES ist das mechanische Gleichgewicht der Mächte ein vorübergehendes, unbeabsichtigtes Merkmal des internationalen Systems. Um das Mächtegleichgewicht als internationale Institution zu etablieren, ging Bull über die Konzeption des zufälligen Mächtegleichgewichts hinaus und beschäftigte sich mit dem Entstehen eines künstlichen Gleichgewichts, in dem Staaten bewusst Aktionen anderer Staaten entgegenwirken, um eine Balance zu erhalten. Das Mächtegleichgewicht sollte aus seiner Sicht nicht als ein mechanisches Arrangement betrachtet werden, das Staaten zu einem bestimmten Verhalten drängt; sondern als ein gemeinsam getroffenes zwischenstaatliches Übereinkommen. Für die ES ist es von besonderer Priorität, dass die Staaten eine einheitliche Ansicht hinsichtlich des Mächtegleichgewichts teilen. ${ }^{36}$ Gerade dieses geteilte Verständnis sowie die daraus entstandenen Regeln konstituieren das Mächtegleichgewicht als eine Institution. Für Waltz ist dieses allen Staaten durch das System zwangsweise auferlegt. Außerdem bestreitet er, dass die Akteure ein gemeinsames Bewusstsein für das Gleichgewicht der Mächte und seine kontinuierliche Ausübung besitzen. ${ }^{37}$

\subsubsection{Macht und Interessen}

In seinem Hauptwerk erläutert Waltz, Macht bedeute, dass ein oder mehrere Staaten die Politik anderer Staaten stärker beeinflussen als jene im Umkehrschluss deren Politik. ${ }^{38}$ Diese Beeinflussung geschehe jedoch nicht immer bewusst. Ein Staat könne das politische Handeln anderer Staaten allein durch seine hervorgeho-

33 Vgl. Schörnig, Neorealismus, aaO. (FN 30), S. 70.

34 Little, The English School's Contribution to the Study of International Relations, aaO. (FN 9), S. 406.

35 Vgl. ebd.

36 Vgl. Alderson / Hurrell, Hedley Bull on International Society, aaO. (FN 20), S. 23.

37 Vgl. Fred Halliday / Justin Rosenberg, »Interview with Kenneth Waltz« in: Review of International Studies, 24, 3 (1998), S. 382.

38 Vgl. Waltz, Theory of International Politics, aaO. (FN 29), S. 192. 
bene Position im internationalen System beeinflussen, ohne die Staaten aktiv zur Beachtung seiner Interessen zu zwingen. Die Neorealisten gehen davon aus, dass die Staaten zugunsten der Sicherheit von einer Machtmaximierung absehen und die Struktur des internationalen Systems den Staaten ein solches Verhalten geradezu aufzwingt. Zugleich bringt das Staatensystem Machtkonstellationen auf internationaler Ebene hervor, die bewaffnete Konflikte verhindern können. ${ }^{39}$ Die Struktur des internationalen Systems löst somit das Machtstreben als zentrales Analysekriterium der klassischen Realisten ab. Hier lassen sich Parallelen zur ES ziehen, allerdings werden bewaffnete Konflikte aus Sicht der ES nicht allein von der Struktur verhindert, sondern aufgrund des selbstbestimmten Willens der Staaten, sich solch einer Struktur unterzuordnen. Der Unterschied liegt darin, dass aus Sicht der ES die Struktur bzw. im Jargon der ES die Regeln der internationalen Gesellschaft aus freien Stücken und aus eigener Überzeugung der Staaten geschaffen wurden, während dies aus Sicht des Neorealismus völlig mechanisch und zufällig geschieht.

\subsubsection{Methodologie}

Waltz entlehnt sein Theorieverständnis dem sozialwissenschaftlichen Positivismus, den Natur- und Wirtschaftswissenschaften. In Hinsicht auf Bulls Kritik am Behaviourismus kann sein Werk als Paradebeispiel für den naturwissenschaftlichen Ansatz gesehen werden, den Bull 1969 in einem polemischen Plädoyer für die traditionalistische Methode radikal verurteilt hat. ${ }^{40}$ Die Debatte zwischen Traditionalisten und Szientisten ist sinnbildlich für die Differenzen zwischen Neorealismus und der ES.

Bei der Entwicklung einer Theorie der IB ist es Waltz zufolge unvermeidlich, die internationale Politik als einen eigenständigen Analysebereich zu betrachten. ${ }^{41}$ Ferner vertritt er den Anspruch, der außenpolitischen Theorie des klassischen Realismus eine systemische Theorie der internationalen Politik entgegenzusetzen. Aus diesem Grunde verschiebt der Neorealismus den Fokus der Untersuchung von der Außenpolitik eines Staates auf die Ebene des internationalen Systems. Er schließt in seinen Erklärungen grundsätzlich von der Struktur des Staatensystems auf das Verhalten von Staaten. Die Neorealisten vertreten somit einen strukturellen Ansatz, der einerseits einen ahistorischen Rahmen liefert und zeitlos auf die Analyse internationaler Systeme angewandt werden kann, andererseits die Disziplin der IB um ein historisches Verständnis der Vergangenheit bringt und somit die Fähigkeit verliert, fundamentale Transformationen des internationalen Systems erklären zu können.

39 Vgl. ebd., S. 102-128.

40 Vgl. Hedley Bull, »International Theory. The Case for a Classical Approach « in: Klaus Knorr / James Rosenau (Hg.), Contending Approaches to International Politics, Princeton 1969, S. 20-38.

41 Vgl. Carlo Masala, Kenneth N. Waltz. Einführung in seine Theorie und Auseinandersetzung mit seinen Kritikern, Baden-Baden 2005, S. 38. In dieser Ansicht schließt sich Waltz Martin Wight an. Dieser fragte bereits 1966 nach einer internationalen Theorie. Siehe Wight, Why is there no International Theory?, aaO. (FN 23). 
Der Neorealismus präsentiert sich als uneinheitliches Theoriegebäude. Durch neorealismusinterne Fortentwicklung und Widerlegung des Waltz'schen Fundaments entsteht bisweilen der Eindruck, dass sich die Neorealisten in ihren Anschauungen nicht immer einig sind. Bei zentralen Entwicklungen der internationalen Politik, wie der zunehmenden Integration Europas oder dem Ende des Ost-West-Konfliktes, weist der Neorealismus zudem eklatante Erklärungsschwächen auf: Er liefert zwar Erklärungsansätze zur Statik des internationalen Systems, für Veränderungen auf subsystemischer Ebene hält der Neorealismus jedoch keine passende Begründung bereit.

Die ES stimmt weitgehend mit der neorealistischen Konzeption des Staatensystems überein. Jedoch widerspricht ihre Ansicht in Bezug auf die Kraft der Struktur: Aus Sicht der ES bleibt trotz strukturellem Druck genügend Handlungsspielraum für die Staaten übrig, da ein Staatensystem zugleich eine Staatengesellschaft umfassen kann. Watson identifiziert diese als»a superstructure, consciously put in place to modify the mechanical workings of the system ${ }^{42}$ Aus dieser Perspektive befindet sich die internationale Gesellschaft auf einer anderen Analyseebene; einer, auf der sich die Untersuchungen nicht auf die Struktur, sondern auf das Level der Institutionen und deren Verfahren fokussieren. Während die ES Veränderungen des Staatensystems als Bedingung für die Entstehung einer internationalen Gesellschaft betrachtet, vollzieht sich eine Transformation des internationalen Systems aus Sicht der Neorealisten nur dann, wenn die Anarchie durch den Aufbau einer Weltregierung überwunden wird. Diese Möglichkeit hält Waltz allerdings für so unwahrscheinlich, dass er sowohl Anarchie als auch den aus ihr resultierenden Selbsthilfecharakter des internationalen Systems zu überzeitlich konstanten Merkmalen erklärt. Aus Sicht der ES fehlt den Neorealisten das gesellschaftliche Element, welches der klassische Realismus hingegen vorweist. Bull beschuldigt Waltz fälschlicherweise davon auszugehen, das internationale System werde im Interesse der internationalen Politik von den Großmächten dominiert. Sein Hauptkritikpunkt ist jedoch die Fokussierung des Neorealismus auf das internationale System. Aus Bulls Perspektive gesehen ist es unmöglich, internationale Beziehungen zu analysieren, ohne dabei die internationale Gesellschaft mit in Betracht zu ziehen. ${ }^{43}$

Im Gegenzug misslang es der ES bisher, eine klare Grenze zwischen internationalem System und internationaler Gesellschaft zu ziehen. Aus diesem Grunde stellt Barry Buzan eine Verbindung zwischen Neorealismus und ES her, indem er den gesellschaftlichen Ansatz der ES mit dem systemischen Zugang der Neorealisten verknüpft. ${ }^{44}$ Durch eine Ausweitung der neorealistischen Konzeption des Staatensystems auf die internationale Gesellschaft der ES könnte ein ausführlicheres und

42 Adam Watson, The Evolution of International Society. A comparative historical analysis, London 1992, S. 311.

43 Vgl. Little, The English School's Contribution to the Study of International Relations, aaO. (FN 9), S. 406-407.

44 Vgl. Barry Buzan, »From International System to International Society: Structural Realism and Regime Theory meet the English School « in: International Organization, 47, 3 (1993), S. 327-352. 
theoretisch fundierteres Verständnis der Staatengesellschaft etabliert sowie ihr Verhältnis zum internationalen System und zur Weltgesellschaft geklärt werden. Von dieser Verknüpfung der ES mit dem Neorealismus profitieren letztlich beide Theorien: Die ES wird aus der Stagnation der historischen Sackgasse befreit, indem das Konzept der Staatengesellschaft einen theoretischen Unterbau erhält. Die Neorealisten werden ihrerseits um wertvolle Kanäle zur Geschichte und zur liberalen Theorie bereichert.

\subsection{Englische Schule vs. Konstruktivismus: Fruchtbare Kooperation}

In den 90er Jahren entstand der Konstruktivismus als Gegenbewegung zu den in dieser Zeit vorherrschenden neorealistischen und neoliberalen Theorien. Dabei stellt er keine kohärente Theorie dar, sondern unterteilt sich in verschiedene Strömungen. ${ }^{45}$ Das Bewusstsein, den Konstruktivismus als eigenständige Theorie zu betrachten, entstand parallel zum Abnabelungsprozess der ES vom Realismus. Der Begriff 'Konstruktivismus< beschreibt Ansätze, welche die intersubjektive Qualität der sozialen Welt und die gegenseitige Konstituierung von Akteur und Struktur betonen und dabei die Rolle von konstitutiven Regeln und Normen sowie die endogene Herausbildung von Interessen und Identitäten in den Vordergrund stellen. Konstruktivisten gehen davon aus, dass sich die Realität nicht unmittelbar ergründen lässt. Die soziale Welt wird vielmehr durch die Art und Weise konstruiert, wie die Menschen untereinander handeln, welche gemeinsamen Vorstellungen existieren und wie die Umwelt auf die Menschen wirkt. ${ }^{46}$ Des Weiteren stimmen alle Konstruktivisten darin überein, dass die Struktur der internationalen Beziehungen sozial angelegt ist. Hier liegt die erste Parallele zur ES: Auch Vertreter der ES schätzen die fundamentalen Strukturen der internationalen Politik vielmehr sozial als materiell ein. Dies zeigt sich allein in der Idee der durch Normen definierten Staatengesellschaft.

\subsubsection{Naturzustand / Menschenbild}

Das Menschenbild der Konstruktivisten lehnt sich an das der ES an: Wendt führt die menschliche Natur auf die Erfüllung der Grundbedürfnisse Nahrung, Wasser, Schlaf und Schutz vor physischer Bedrohung zurück. Danach geht es um ontologische Sicherheit und eine stabile Umwelt, die Natur und die soziale Welt betreffend. Der Mensch wird als 'zoon politikon< begriffen, als ein soziales Wesen, das nach Vergesellschaftung trachtet. Er bedarf der Fähigkeit, seine Lebensbedingungen eigenhändig zu entwickeln und zu verbessern. ${ }^{47}$ Mit diesen Punkten stimmt das Menschenbild der ES überein.

45 Seine Hauptvertreter sind Alexander Wendt, John Gerard Ruggie, Friedrich Kratochwil, Nicholas Onuf und Peter Katzenstein.

46 Vgl. Cornelia Ulbert, »Sozialkonstruktivismus« in: Siegfried Schieder / Manuela Spindler (Hg.): Theorien der Internationalen Beziehungen, Opladen 2003, S. 391.

47 Vgl. Alexander Wendt, Social Theory of International Politics, Cambridge 1999, S. 131-132.

ZfP 55. Jg. 3/2008 
Wendt bestätigt die neorealistische Annahme, dass Anarchie das entscheidende strukturelle Ordnungsprinzip ist, das für Staaten im internationalen System handlungsleitend wirkt. Im Gegensatz zu den Neorealisten geht Wendt jedoch davon aus, dass Anarchie ein gegebenes Faktum ist, welches unmittelbar das Handeln der Staaten bestimmt. Seiner Meinung nach ist Anarchie»what states make of it «48 und kann demzufolge vielfältige Ausprägungen haben. Er unterscheidet zwischen drei Systemstrukturen: In Anlehnung an die Theorietraditionen der ES differenziert er zwischen Hobbes'scher, Locke'scher und Kantianischer Struktur. ${ }^{49}$ Diese verkörpern wie bei der ES verschiedene Grade an Normen. Welche Art von Struktur realisiert wird, hängt dabei zum einen vom Maß an Internalisierung der Normen, zum anderen vom Maß an Kooperation ab: Inwieweit Normen von den jeweiligen Akteuren akzeptiert werden, lässt sich an der Motivation der Akteure zur Einhaltung der Regeln ablesen: Entweder befolgen sie Normen aus einem Zwang heraus, weil es in ihrem Interesse liegt oder weil sie diese als legitim ansehen. ${ }^{50}$ Das Maß an Kooperation ist auch für die ES ein entscheidender Faktor. Es schlägt sich darin nieder, dass es einen wachsenden Anteil an geteilten Ideen und Interessen gibt, die wiederum zur Herausbildung der internationalen Gesellschaft führen. ${ }^{51}$

\subsubsection{Form der internationalen Beziehungen}

Der Konstruktivismus betont, parallel zur ES, den gesellschaftlichen Charakter der internationalen Beziehungen. Diese sind demgemäß»deeply social, [...] a realm of action in which the identities and interests of states and other actors are discursively structured by intersubjective rules, norms and institutions.$^{52}$ Den Konstruktivisten zufolge ist die Staatengesellschaft kein objektives Faktum und somit Resultat einer bestehenden Struktur, sondern eine gesellschaftliche Struktur, die aus der Folge eines Prozesses heraus entstanden ist. Analog zur ES messen sie die Bedeutung des internationalen Systems oder der internationalen Gesellschaft an der Beschaffenheit der zwischenstaatlichen Interaktionen. ${ }^{53}$ Die konstruktivistische Definition der internationalen Gesellschaft, derzufolge»the international system is a society< in which states, as a condition of their participation in the system, adhere to shared norms and rules in a variety of issue areas « ${ }^{54}$, könnte ebenso von einem Vertreter der

48 Alexander Wendt, »Anarchy is what states make of it. The social construction of power politics « in: International Organization, 46, 2 (1992), S. 391.

49 Vgl. Wendt, Social Theory of International Politics, aaO. (FN 47), S. 247. Siehe dazu Wight, International Theory, aaO. (FN 6).

50 Vgl. Wendt, Social Theory of International Politics, aaO. (FN 47), S. 250.

51 Vgl. ebd., S. 254.

52 Christian Reus-Smit, »Imagining society: constructivism and the English School in: British Journal of Politics and International Relations, 4, 3 (2002), S. 488.

53 Vgl. ebd., S. 489.

54 Ronald L. Jepperson / Alexander Wendt / Peter J. Katzenstein, »Norms, identity, and culture in national security « in: Peter J. Katzenstein (Hg.), The Culture of National Security. Norms and Identity in World Politics, New York 1996, S. 45. 
ES stammen. Für beide Denkschulen ist der Prozess des sozialen Lernens genauso bedeutend wie die spezifische gegenseitige Abhängigkeit, da sowohl die internationale Gesellschaft durch die Staaten als umgekehrt die Staaten durch die internationale Gesellschaft geformt werden. ${ }^{55}$

Der Konstruktivismus nimmt den Wandel der Identitäten und Interessen der Staaten als Ausgangspunkt. Der Staat steht folglich im Zentrum der Untersuchung. Seine Entwicklung wird als Ergebnis von Interaktionen in einem breiten institutionellen Umfeld angesehen, welches auf Normen und daraus folgenden Strukturen aufgebaut ist. Die Institutionen sind sozusagen Antriebskräfte, die als Resultat der normativen Vergesellschaftung eine gewisse Anzahl an Regeln, Normen und Prinzipien erlassen. Gleichzeitig sind internationale Institutionen in der Lage, staatliche Identitäten und Interessen zu verändern. Im Gegensatz zur ES, die Identitäten und ihre Interessen als fix und unveränderlich wahrnimmt, eröffnen die Konstruktivisten hier neue Wege, indem sie auf die Transformation der Interessen hinweisen. ${ }^{56}$ In Übereinstimmung mit der ES bestätigen die Konstruktivisten die Existenz einer internationalen Ordnung. Auch wenn im internationalen System keine zentrale Ordnungsinstanz existiert, so sind zahlreiche Institutionen vorhanden, welche die Interaktionen zwischen den Staaten regeln.

Differenzen zwischen beiden Theorieschulen gibt es in der Handhabung ungleicher Machtverteilungen im internationalen System, darin einschließend Probleme der Entstehung und des Zerfalls von Großmächten, der Institution Großmächte sowie des Pluralismus der Kulturen. ${ }^{57}$ Des Weiteren unterscheiden sich Konstruktivismus und ES in der regionalen Ausrichtung: Während die ES den überregionalen Charakter der internationalen Gesellschaft betont, gehen die Konstruktivisten davon aus, dass der Übergang von einer internationalen Gesellschaft zur Weltgesellschaft nur zwischen bestimmten Staaten geschehen kann und begrenzt ist auf eine kulturelle Region.

\subsubsection{Macht und Interessen}

Die Konstruktivisten gehen von der These aus, dass das Handeln der Akteure, d.h. der Staaten, sich nicht nach materiellen Interessen und Machtmaximierung, sondern nach Normen und Werten orientiert. Ihr Hauptaugenmerk liegt auf der Entstehung und Wirkung von Normen in den internationalen Beziehungen. Zur Erklärung des Wandels innerhalb der internationalen Politik ist dabei insbesondere die Herausbildung von Identitäten und Interessen grundlegend: Die Identitätsbildung ist ein Prozess der sozialen Definition von Seiten der Akteure, der auf kollek-

55 Vgl. Timothy Dunne, »International Society: Theoretical Promises Fulfilled?« in: Cooperation and Conflict, 30 (1995), S. 146.

56 Vgl. Janine Kissolewski, "Norms in international society. English School meets constructivists « presented at: BISA Annual Conference, Bradford, Dezember 2000, S. 8.

57 Vgl. Jacek Czaputowicz, »The English School of International Relations and its Approach to European Integration« in: Studies E Analyses, 2, 2 (2003), S. 29. 
tiven Vorstellungen dieser über sich selbst und die Welt basiert. Ihre Interessen legen Akteure fest, während sie ihre Stellung innerhalb der internationalen Politik definieren. Hierbei werden die Institutionen der internationalen Beziehungen als Struktur der Interessen und Identitäten erfasst. Dementsprechend bedingen sich Identitäten und Institutionen gegenseitig.

Die drei Hauptströmungen bei der Betrachtung des Interesses aus kontruktivistischer Sicht sind die sschool of norms and cultures, sschool of soft rationalism< und ssystemic schook. Alle drei Schulen verfolgen eine unterschiedliche Methode, Herkunft und Entstehung von Interessen zu definieren. Erstere versteht Interessen als Folge kultureller Konditionierung. Die zweite betrachtet Interessen abhängig davon, wie deren Urheber politische Kausalität verstehen. Die dritte lehnt sich an das Interessenverständnis der ES an und leitet Interessen von den Identitäten der Akteure ab, die wiederum davon abhängen, welche Funktion die Akteure innerhalb des globalen Systems innehaben. ${ }^{58}$

\subsubsection{Methodologie}

Konstruktivistische Ansätze gehen von der Konstruktion der sozialen Welt aus, mit der erkenntnistheoretische Orientierungen einhergehen, beruhend auf der Einsicht, dass auch Wissen sozial konstruiert wird. Ziel konstruktivistischer Untersuchungen ist letztlich, mit Hilfe verschiedener Methoden erklären zu können, wie diese Konstruktionen erzeugt werden. Wendt bewegt sich wie Kenneth Waltz auf der systemischen Ebene und versucht, Phänomene der internationalen Beziehungen aus der Struktur des internationalen Systems heraus zu erklären. Beide verfolgen somit einen strukturalistischen Ansatz. Der entscheidende Unterschied zu Waltz besteht darin, dass für Wendt nicht materielle, sondern soziale Faktoren bei der Analyse der internationalen Politik relevant sind.

Eine einheitliche Methodologie des Konstruktivismus gibt es nicht. Frühe Konstruktivisten bestanden darauf, dass die Analyse der Ideen, Kultur und Normen eine interpretative Methodik erfordert. ${ }^{59}$ Wendt widmet sich dagegen der Haltung des szientistischen Neorealismus, der einen positivistischen und sozialwissenschaftlichen Ansatz verfolgt. Im Gegensatz zur ES ist die historische Analyse folglich kein fester Bestandteil konstruktivistischer Lehre. Die Konstruktivisten tendieren vielmehr zur Übernahme der sozialwissenschaftlichen Methodik und geben dieser eine interpretierende Wendung. ${ }^{60}$

Parallel zur via media der ES gibt es bei den Konstruktivisten einen middle ground, dessen Motivation darin liegt, sowohl zwischen den jeweiligen Rändern im

58 Auch Ernst Haas betont die Affinität der ssystemic schook zur ES, insbesondere zu Bull und Buzan. Siehe Ernst B. Haas, DDoes Constructivism subsume Neofunctionalism? « in: Thomas Christiansen / Knud Eric Jørgensen / Antje Wiener (Hg.), The Social Construction of Europe, London 2001, S. 26-27.

59 Siehe Friedrich Kratochwil, »Regimes, Interpretation and the >Science< of Politics: A Reappraisal« in: Millennium, 17, 2 (1988), S. 263-284.

60 Alderson / Hurrell, Hedley Bull on International Society, aaO. (FN 20), S. 37. 
rationalistischen und konstruktivistischen Lager, als auch zwischen modernen und postmodernen Varianten des Konstruktivismus Brücken zu bauen und einen Mittelweg zwischen unterschiedlichen, teilweise unvereinbar scheinenden theoretischen Positionen einzuleiten.

Die Gemeinsamkeiten der beiden Denkschulen sind offensichtlich. Sowohl der Konstruktivismus als auch die ES heben die zentrale Bedeutung von Normen in der internationalen Politik hervor und gehen von der Vorstellung der internationalen Gesellschaft aus. Beide Denkschulen überschneiden sich in der Theorie des Naturzustands und der Bestimmung menschlicher Grundbedürfnisse. Darüber hinaus konzentrieren sie sich auf den Staat als zentralen Akteur und dessen Rolle im internationalen System und erklären Veränderungen in der internationalen Politik durch das Prisma der Kultur. Als Konsequenz daraus akzeptieren sie die Beeinflussung der Staaten durch die Struktur, die dennoch Handlungsspielraum für Verbesserungen der internationalen Beziehungen zulässt. ${ }^{61}$ Dunne geht sogar soweit zu behaupten, die ES könne exemplarisch für den Konstruktivismus angesehen werden. ${ }^{62}$ Auch Wendt ordnet die ES den konstruktivistischen Theorien zu. ${ }^{63}$ Manche Werke der ES, insbesondere Mannings >The Nature of International Society<, könnten als konstruktivistische Beispiele betrachtet werden, auch unter dem Vorbehalt, dass zu dieser Zeit die konstruktivistische Schule noch gar nicht etabliert war. Im Umkehrschluss beziehen sich fast alle Konstruktivisten in ihren Ausführungen auf Bull und andere Autoren der ES, Wendt gibt sogar zu, seine Sprache an die von Martin Wight und der ES anzupassen. ${ }^{64}$

Die beiden Theorien haben allerdings nicht nur Gemeinsamkeiten, sondern mindestens ebenso viele Unterschiede: Einer davon ist der Zugang zur Geschichte. Während die ES an der historischen Erforschung der internationalen Gesellschaft festhält, ist der Historizismus bei den Konstruktivisten wenig ausgeprägt. Dafür beschäftigt sich die ES im Gegensatz zum Konstruktivismus nicht mit der Frage, in welchem Ausmaß Anarchie Kooperation ermöglicht, sondern sucht nach einem Weg, anarchische Strukturen zu überwinden. Eine weitere Diskrepanz ist die Unantastbarkeit der staatlichen Souveränität, die für Bull im Gegensatz zu den Konstruktivisten kein Objekt der Transformation darstellt. Die ES fokussiert sich auf die wechselseitige Beeinflussung von Menschenrechten und Staatengesellschaft, während sich die Konstruktivisten auf den Einfluss der Menschenrechte auf die zwischenstaatlichen Beziehungen konzentrieren. ${ }^{65}$

61 Vgl. Hidemi Suganami, »Alexander Wendt and the English School « in: Journal of International Relations and Development, 4, 4 (2001), S. 406-411.

62 Vgl. Timothy Dunne, »The Social Construction of International Society « in: European Journal of International Relations, 1, 3 (1995), S. 367-389.

63 Gleichwohl stellt er fest:»The English School does not explicitly address state identity formation, but does treat the international system as a society governed by shared norms «. Siehe Wendt, Social Theory of International Politics, aaO. (FN 47), S. 31.

64 Vgl. ebd., S. 247.

65 Vgl. Reus-Smit, Imagining society, aaO. (FN 52), S. 501-502. 
Prinzipiell können sich beide Theorien gewinnbringend ergänzen: Die konstruktivistische Theorie ist besonders geeignet, Erklärungen für verschiedene konkrete Situationen zu liefern und wird daher häufig zur Analyse der Europäischen Integration angewandt, während die ES bewährter im Begründen allgemeinen Wandels und Veränderungen ist und breiter auf ethische Probleme eingeht. Der Konstruktivismus könnte sich in dieser Hinsicht vom normativen Ansatz der ES inspirieren lassen und der ES im Gegenzug durch Anwendung empirischer Konzepte eine soziologische Komponente beifügen.

Durch Übernahme der konstruktivistischen Sprache, die präziser und szientistischer als die der ES ist, würde die theoretisch oberflächliche Trennung von internationaler Gesellschaft und Weltgesellschaft sowie die daraus folgende Zweiteilung in Pluralismus und Solidarismus eine eindeutige theoretische Auslegung erhalten. Eine Verbindung der ES mit konstruktivistischen Überlegungen könnte auf diese Weise zur Lösung der Pluralismus-Solidarismus-Frage beitragen. Christian Reus-Smit warnt jedoch beide Denkschulen vor einer unbedachten Übernahme der jeweiligen Stereotype der anderen, was seiner Meinung nach größtenteils zur Behinderung eines produktiven Dialogs führe. ${ }^{66}$ Dennoch glaubt er, dass die Verknüpfung des empirischen mit dem normativen Ansatz wesentlich bei der Analyse internationaler Handlungen ist. So können Interessen nicht ohne Werte definiert und Strategien nicht ohne Erfahrungswissen entwickelt werden. Aufgrund ihrer jeweiligen Grenzen sind sowohl die ES als auch der Konstruktivismus per se nicht in der Lage, den Standard einer auf die Praxis anwendbaren Theorie zu erreichen. Werden nunmehr Elemente der beiden Theorien verbunden, könnte dies sowohl den Konstruktivismus als auch die $\mathrm{ES} » \mathrm{closer}$ to this ideal $\ll^{67}$ bringen.

\section{Standortbestimmung der Englischen Schule}

Es gibt drei deutliche Unterschiede zwischen der ES und dem klassischen Realismus bzw. dem Neorealismus: Erstens widerspricht die ES der Annahme der Realisten, dass Hobbes' Kriegszustand eines jeden gegen jeden auf die internationale Ebene übertragbar ist. Der ES zufolge sind Staaten weniger verwundbar als Individuen und haben weniger Angst vor einem plötzlichen Tod. Sie sind ungleich im Besitz von Macht und Ressourcen und versuchen weniger, sich gegenseitig zu zerstören, als im Naturzustand lebende Menschen. Darüber hinaus sind Staaten fähig, Regeln der Koexistenz aufzustellen, basierend auf Eigeninteresse und rationaler Vorsicht. ${ }^{68}$ Zweitens wird Normen und Institutionen von Seiten des klassischen Realismus und insbesondere des Neorealismus zu wenig Beachtung geschenkt. Ordnung basiert häufig auf einem Mächtegleichgewicht, hierarchischen Strukturen und Zwang. Dabei erscheint der Begriff >Gesellschaft < dürftig - vor allem aus Sicht des Schwächeren. Gleichwohl ist der Begriff gerechtfertigt, und zwar in dem Ausmaß, in dem

66 Vgl. ebd., S. 488.

67 Ebd., S. 506.

68 Vgl. Bull, The Anarchical Society, aaO. (FN 2), S. 44-49. 
solch eine erzwungene machtpolitische Ordnung auf einer gemeinsamen Grundlage an Vereinbarungen und gegenseitig anerkannten Interessen beruht. Denn der zentrale Punkt der Definition der internationalen Gesellschaft ist die hervorgehobene Bedeutung gemeinsamer Normen und Regeln sowie die Beachtung der Beziehungen der Staaten untereinander. ${ }^{69}$ Das dritte divergierende Merkmal der ES und der Realisten betrifft die Natur des internationalen Systems und dessen historische Entwicklung. Die ES teilt die realistische Betonung des Staatensystems und die Notwendigkeit einer Analyse auf Ebene des internationalen Systems. Entsprechend der ES darf das internationale System dabei aber nicht als dezentralisierte, anarchische Struktur betrachtet werden, in dem sich funktional undifferenzierte Akteure nur in Bezug auf die Machtverteilung unterscheiden. Aus ihrer Sicht entwickelte sich das Staatensystem im Laufe der Geschichte durch gemeinsame Regeln und Normen sowie wechselseitige Erwartungen zu einer internationalen Gesellschaft. Die Konzepte der Staatssouveränität, das Völkerrecht sowie der Krieg entstanden demnach nicht» by the game of power politics «70: Das Staatensystem ist Resultat eines historischen Prozesses und kann nicht mittels zeitloser unveränderlicher Gesetze eruiert werden, wie dies bei Morgenthau, aber vor allem in den ahistorischen Abstraktionen von Waltz zu beobachten ist.

Die ES betrachtet Institutionen der internationalen Gesellschaft als durch Normen und Werte kulturell determiniert sowie Interessen und Identitäten als vorgegeben und unveränderlich. Damit setzt sie sich selbst Grenzen in der Analyse des strukturellen Wandels innerhalb der internationalen Gesellschaft. An dieser Stelle könnten die Konstruktivisten eingreifen. Der Konstruktivismus befasst sich insbesondere mit dem Wandel von Identitäten und Interessen innerhalb der Staatengesellschaft und untersucht die Konstruierung von Identitäten durch die internationale Gesellschaft. ${ }^{71}$

Ole Wæver untersucht die Unterschiede zwischen den Theorien der IB anhand von sechs Kriterien: Materialistisch versus sozial konstruierte internationale Beziehungen; Funktion der Ideengeschichte; Rolle der Forscher; Erkenntnistheorie; Konzept der Sprache und Theorie sowie die Rolle von Tradition in den internationalen Beziehungen. Nach dem ersten Kriterium kam Wæver zu dem Ergebnis, die ES in der Mitte zwischen dem klassischen Realismus und dem Konstruktivismus anzusiedeln. Auf der einen Seite sind soziale Beziehungen für die ES mit Abstand bedeutender als für Realisten, auf der anderen Seite ist die ES jedoch materialistischer als die konstruktivistische Schule. In Anbetracht der anderen Kriterien lokalisiert Wrever die ES näher dem klassischen Realismus als dem Konstruktivismus. ${ }^{72}$

69 Vgl. Alderson / Hurrell, Hedley Bull on International Society, aaO. (FN 20), S. 23.

70 Ebd., S. 24.

71 Vgl. Kissolewski, Norms in international society, aaO. (FN 56), S. 2.

72 Siehe Ole Wrever, "Does the English School's Via Media equal the Contemporary Constructivist Middle Ground?, Or: On the difference between philosophical scepticism and sociological theory « presented at: BISA Annual Conference, Manchester, Dezember 1999, S. 1. 
Einordnung der Englischen Schule in die Theorien IB

\begin{tabular}{|lll|}
\hline Neorealism & Classical Realism & English school \\
\hline Materialist & & Constructivism \\
\hline
\end{tabular}

Quelle: In Anlehnung an Ole Wrever, »Does the English School's Via Media equal the Contemporary Constructivist Middle Ground?, Or: On the difference between philosophical scepticism and sociological theory « presented at: BISA Annual Conference, Manchester, Dezember 1999, S. 7.

Die Vorstellung der Weltgesellschaft entfernt die ES am weitesten von den Neorealisten und rückt den klassischen Realismus näher in Richtung der ES. Mit diesem verbindet die ES zudem eine ähnliche Methodik. Mit dem Konstruktivismus teilt die ES die Vorstellung der internationalen Gesellschaft sowie die Betonung von Normen und Werten, unterscheidet sich jedoch in der methodischen Ausrichtung. Unter Berücksichtigung dieser Kriterien kann die ES in der Mitte zwischen dem klassischen Realismus und dem Konstruktivismus positioniert werden - veranschaulicht wird das Ergebnis in folgender Tabelle:

Neorealismus, klassischer Realismus, Englische Schule und Konstruktivismus im Vergleich

\begin{tabular}{|l|l|l|l|l|}
\hline & Neorealismus & $\begin{array}{l}\text { Klassischer } \\
\text { Realismus }\end{array}$ & $\begin{array}{l}\text { Englische } \\
\text { Schule }\end{array}$ & $\begin{array}{l}\text { Konstruk- } \\
\text { tivismus }\end{array}$ \\
\hline $\begin{array}{l}\text { Natur- } \\
\text { zustand }\end{array}$ & $\begin{array}{l}\text { Anarchie, } \\
\text { Selbsthilfe- } \\
\text { system }\end{array}$ & $\begin{array}{l}\text { Anarchie, } \\
\text { Kampf ums } \\
\text { Überleben }\end{array}$ & $\begin{array}{l}\text { Anarchie, egois- } \\
\text { tischer, jedoch } \\
\text { vernunftbegab- } \\
\text { ter Mensch }\end{array}$ & $\begin{array}{l}\text { Unterschied- } \\
\text { liche Anar- } \\
\text { chieformen, } \\
\text { Mensch } \\
\text { trachtet nach } \\
\text { Vergesell- } \\
\text { schaftung }\end{array}$ \\
\hline $\begin{array}{l}\text { Form der } \\
\text { internati- } \\
\text { onalen Be- } \\
\text { ziehungen }\end{array}$ & $\begin{array}{l}\text { Internationa- } \\
\text { les System, } \\
\text { Vorstellung } \\
\text { der internatio- } \\
\text { nalen Gesell- } \\
\text { schaft } \\
\text { unmöglich }\end{array}$ & $\begin{array}{l}\text { Internationales } \\
\text { System, Vorstel- } \\
\text { lung der inter- } \\
\text { nationalen } \\
\text { Gesellschaft } \\
\text { möglich }\end{array}$ & $\begin{array}{l}\text { Internationale } \\
\text { Gesellschaft }\end{array}$ & $\begin{array}{l}\text { Internationale } \\
\text { Gesellschaft, } \\
\text { regional be- } \\
\text { grenzt }\end{array}$ \\
\hline
\end{tabular}




\begin{tabular}{|l|l|l|l|l|}
\hline & Neorealismus & $\begin{array}{l}\text { Klassischer } \\
\text { Realismus }\end{array}$ & $\begin{array}{l}\text { Englische } \\
\text { Schule }\end{array}$ & $\begin{array}{l}\text { Konstruk- } \\
\text { tivismus }\end{array}$ \\
\hline Interessen & $\begin{array}{l}\text { Verfolgung } \\
\text { des egoisti- } \\
\text { schen Eigen- } \\
\text { interesses }\end{array}$ & $\begin{array}{l}\text { Verfolgung des } \\
\text { egoistischen Ei- } \\
\text { geninteresses }\end{array}$ & $\begin{array}{l}\text { Durch Normen } \\
\text { und Werte defi- } \\
\text { niert }\end{array}$ & $\begin{array}{l}\text { Durch Nor- } \\
\text { men und Wer- } \\
\text { te definiert }\end{array}$ \\
\hline $\begin{array}{l}\text { Metho- } \\
\text { dologie }\end{array}$ & $\begin{array}{l}\text { Positivismus, } \\
\text { am Muster der } \\
\text { Naturwissen- } \\
\text { schaften ange- } \\
\text { lehnt }\end{array}$ & $\begin{array}{l}\text { Normative The- } \\
\text { orie / Bezug zu } \\
\text { historischen } \\
\text { Vordenkern }\end{array}$ & $\begin{array}{l}\text { Interpretativer } \\
\text { Ansatz / Histo- } \\
\text { rizismus }\end{array}$ & $\begin{array}{l}\text { Soziologische } \\
\text { Theorie }\end{array}$ \\
\hline
\end{tabular}

Eigene Darstellung

\section{Fazit}

Mit den Worten von Buzan:»The English School [...] is ripe for reconsideration. ${ }^{73}$ Sie legte ihr Image als >englischer Patient ${ }^{74} \mathrm{ab}$ und ist auf einem guten Weg in eine dritte Generation aktiver Forschung und Lehre. Nicht länger stellt die ES eine Abspaltung des Realismus dar, sondern nimmt zwischen den Konstruktivisten und den klassischen Realisten einen eigenen Platz innerhalb der Theorien der IB ein. Dennoch steht sie bis heute abseits der amerikanischen Mainstream-Theorien und ist anfällig für Anschuldigungen, stagnierend und rückständig zu sein. Gleichwohl ist es an der Zeit, dem historischen, konstruktivistischen, multiparadigmatischen und methodologisch pluralistischen Ansatz der ES mehr Prominenz innerhalb der IB einzuräumen. Dieser ebnet Wege, eine Anzahl an bestehenden Problemen der IB in Angriff zu nehmen: Insbesondere der Historizismus sowie der methodische Pluralismus bieten Möglichkeiten, nicht nur die fragmentartige Welt der Theorien der IB zu vergemeinschaftlichen, sondern auch die Kommunikation zwischen den IB, anderen Sozialwissenschaften und der Weltgeschichte zu intensivieren. Die Theorien der IB haben viel von anderen Disziplinen entliehen, bisher jedoch wenig an diese zurückgegeben. Um dieses Ungleichgewicht auszubalancieren, sollten sie Konzepte unterstützen, die von anderen Disziplinen in deren Debatten integriert werden könnten. Die ES liefert solche Konzepte und aus Sicht Buzans ebenso das Potential» of reviving grand theory $« .{ }^{75}$

73 Barry Buzan, »The English School as a Research Program: an overview, and a proposal for reconvening " presented at: BISA Annual Conference, Manchester, Dezember 1999, S. 2.

74 Vgl. Ian Hall, "Still the English patient? Closures and inventions in the English school« in: International Affairs, 77, 3 (2001), S. 931-942 sowie Barry Buzan / Richard Little, "The >English patient< strikes back: a response to Hall's mis-diagnosis « in: International Affairs, 77, 3 (2001), S. 943-946.

75 Barry Buzan, »The English School: an underexploited resource in IR « in: Review of International Relations, 27 (2001), S. 488. 
Die Bedeutung der ES liegt folglich nicht in der Entwicklung einer bestimmten Forschungsmethode, sondern darin, verschiedene Disziplinen - Geschichte, Völkerrecht, Soziologie, Politische Philosophie - in das Studiums der IB zu integrieren. Aufgrund ihrer vom Realismus abweichenden Auslegung der Entstehungsgeschichte der internationalen Gesellschaft und ihrer eigenen Interpretation der internationalen Beziehungen stellt sie eine Alternative zum klassischen Realismus dar. Während die Realisten von einem konstanten, unveränderlichen internationalen System ausgehen, weisen Vertreter der ES darauf hin, dass in der Geschichte verschiedene Formen des internationalen Systems existierten. Diese Formen wandelten sich von unabhängigen Staaten zu zentralisierten Imperien und nahmen zuweilen die Gestalt einer internationalen Gesellschaft an. Dank dieser Beobachtung wissen wir heute, dass unser gegenwärtiges System der Nationalstaaten nicht definitiv ist und in $\mathrm{Zu}$ kunft weiteren Transformationen unterliegen kann.

In vielerlei Hinsicht ist das Denken der ES sicherlich unterentwickelt. In einigen Aspekten hat sie sich bisher nicht über den klassischen Realismus hinaus entfaltet. Andererseits hat sie aber auch keine Rückschritte zu verbuchen. Die Schlüsselansichten des klassischen realistischen Denkens wiederholen sich alle in der ES, dennoch lassen sich die Mitglieder der ES auch von anderen Theorien inspirieren. Die Neorealisten dagegen fokussieren sich nur auf eine Theorie, den klassischen Realismus. Durch diese einseitige Betrachtungsweise erstarrt die neorealistische Theorie in ihren Auslegungen und verfälscht dadurch die Realität der internationalen Politik. Was den Realisten fehlt, ist der ausgeprägte interpretative Ansatz der ES: Die Betrachtung der internationalen Beziehungen aus historischer Perspektive verdeutlicht, dass der Anarchie eine eher unübliche Struktur innewohnt. Diese war stets fragil und ebnete der Hierarchie fast ausnahmslos den Weg. Die Realisten übersehen dies und gehen davon aus, dass Anarchie eine robuste Struktur besitzt, die nicht explizit erklärt werden muss. Insofern präsentiert die ES durch den Rückblick in die Weltgeschichte eine ausführliche Analyse dessen, was die Realisten bisher versäumten.

Am Ende bleibt die Frage, inwiefern die Theorien der IB Antworten auf zukünftige Veränderungen in der internationalen Politik geben. Sind sie nicht vielmehr statische Theorien ihrer Zeit? Die ES geht beispielsweise von der Prämisse aus, dass der pluralistische Rahmen ihr ermöglicht, alle in der Weltgeschichte auftretenden Erscheinungen internationaler Beziehungen zu analysieren. Der Realismus würde wahrscheinlich dieselbe Behauptung aufstellen. Doch gestaltet sich bereits die Untersuchung der Gegenwart, der Zeit nach dem Kalten Krieg, für beide Theorien als schwierig. Die Realisten vertreten eine Theorie, die das Verhalten von Großmächten erklärt. Heutzutage ist die internationale Politik jedoch durch Unipolarität gekennzeichnet, d.h. mit den Vereinigten Staaten existiert gegenwärtig nur eine Großmacht. Dies berücksichtigt jedoch weder der Realismus noch die ES. In beiden Theorien ist das Mächtegleichgewicht zentral. Wie sich dies in einer unipolaren Staatengesellschaft verhält, sollte indessen überdacht werden. Folgerichtig ist für alle Theorien der IB unerlässlich, sich dem Wandel der Zeit anzupassen, Lücken zu schließen und sich fruchtbaren Kooperationen mit anderen Denkschulen zu öffnen. 


\section{Zusammenfassung}

Konfrontation oder Kooperation? In der Theorienlandschaft der IB konkurrieren die verschiedensten Weltanschauungen um gegenseitige Anerkennung. Die Englische Schule (ES) fokussiert sich auf die internationale Gesellschaft, die Überwindung von Anarchie in der internationalen Politik und die Herstellung internationaler Ordnung. Aus ihrer Sicht können anarchische Verhältnisse durch normative Integration der Staatenbeziehungen im Zaum gehalten werden. Mit dieser Annahme löst sich die ES, die lange Zeit als Randgruppe des klassischen Realismus gehandelt wurde, von der realistischen Theoriebildung und übernimmt einen eigenen Standort innerhalb der IB. Dieser Beitrag ordnet die ES als eigenständige Größe fest in das Theoriengefüge der internationalen Politik ein. Anhand von vier Kriterien wird die ES mit dem klassischen Realismus, dem Neorealismus und dem Konstruktivismus verglichen sowie Chancen und Potentiale einer wechselseitigen Befruchtung der Denkschulen untereinander aufgezeigt.

\section{Summary}

Confrontation or cooperation? In the theoryscenery of IR different ideas compete for mutual recognition. The members of the English School (ES) focus on international society, the questions of overcoming anarchy in international politics and maintaining international order. From their point of view anarchical conditions could be contained by normative integration of the relations between states. With this assumption the ES, which a long time was considered as a marginal group of classical realism, disengages from realistic theory and takes up an own position within the theories of IR. This article classifies the ES as a self-contained school of thought. On the basis of four criterions the ES will be compared with classical realism, neorealism and constructivism. Furthermore the article presents prospects and potentials of cross-fertilization within the different theories.

Andrea Gebhardt, The English School meets theories of international relations A statement of place 